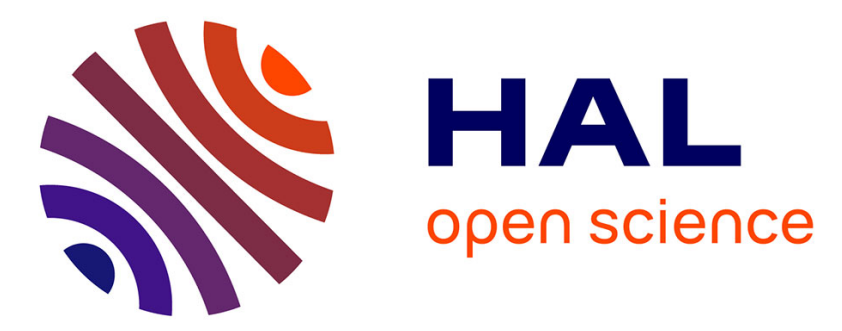

\title{
A Landmark-Based Approach for Robust Estimation of Exposure Index Values in Digital Radiography
}

\author{
Paolo Irrera, Isabelle Bloch, Maurice Delplanque
}

\section{To cite this version:}

Paolo Irrera, Isabelle Bloch, Maurice Delplanque. A Landmark-Based Approach for Robust Estimation of Exposure Index Values in Digital Radiography. Medical Image Computing and Computer-Assisted Intervention - MICCAI 2015 - LNCS 9350, Oct 2015, Munich, Germany. 10.1007/978-3-319-245713_75. hal-01218476

\author{
HAL Id: hal-01218476 \\ https://hal.science/hal-01218476
}

Submitted on 22 Oct 2015

HAL is a multi-disciplinary open access archive for the deposit and dissemination of scientific research documents, whether they are published or not. The documents may come from teaching and research institutions in France or abroad, or from public or private research centers.
L'archive ouverte pluridisciplinaire HAL, est destinée au dépôt et à la diffusion de documents scientifiques de niveau recherche, publiés ou non, émanant des établissements d'enseignement et de recherche français ou étrangers, des laboratoires publics ou privés. 


\title{
A landmark-based approach for robust estimation of exposure index values in digital radiography
}

\author{
Paolo Irrera $^{1,2}$, Isabelle Bloch ${ }^{1}$, Maurice Delplanque $^{2}$ \\ 1 Institut Mines Telecom, Telecom ParisTech, CNRS LTCI, Paris, France \\ 2 EOS imaging, Paris, France
}

\begin{abstract}
The exposure index (EI) gives a feedback to radiographers on the image quality in digital radiography, but its estimation on clinical images raises many challenges. In this paper we provide a critical overview of state of the art methods that address this problem and we show that more robust results can be obtained by detecting anatomical structures. This new approach implicitly manages the presence of multiple structures in the field-of-view. Moreover, we propose a landmarkbased method that, by exploiting redundancy of local estimates, is more robust to potential detection errors.
\end{abstract}

Keywords: Exposure index, anatomical structures detection, local estimates, full-body X-ray imaging

\section{Introduction}

The exposure index (EI) is a standardized image quality measure for $2 \mathrm{D}$ digital radiography (DR) [1] that expresses the amount of signal level reaching the detector and is proportional to the squared signal to noise ratio [2]. Therefore, it can be used to define the as low as reasonably achievable (ALARA) amount of dose for an exam according to the associated medical purpose. While the physical meaning of the EI has been extensively justified, how to correctly define it on clinical images has been less addressed. Only the attenuated X-rays relevant for the diagnosis need to be taken into account, which in practice requires to define a region of interest (ROI). In this paper, we show that, by detecting anatomical structures of interest, the estimated EI values are more consistent compared with those obtained with methods suggested in the literature [3]. Moreover, we propose to compute multiple local EI estimates associated with landmarks that belong to a given structure of interest. The regional EI is then computed as a weighted mean of the local estimates. This paper also addresses the problem due to the presence of different anatomical structures in the field-of-view. In this case, the method should be capable of computing an EI value for each structure to quantify image quality according to the most relevant anatomical region for the diagnosis. Our experiments are performed on images acquired with EOS system, that is dedicated to the full-body analysis of the musculoskeletal 
apparatus [4]. However, the discussion and the method proposed in this paper can be generalized to other DR systems.

The paper is organized as follows. Section 2 overviews the theoretical background. Section 3 introduces the proposed approaches. Section 4 presents the results. Finally, Section 5 summarizes the contributions and perspectives.

\section{Exposure index algorithm}

\subsection{Overview}

The EI is computed from the raw image $\mathbf{q}$ that is processed to correct imperfections of the detector and geometric distortions. Other image processing steps such as noise reduction and contrast enhancement must be avoided because they significantly influence the image quality. Then, a subset $\Psi$ of the whole pixel space $\Omega$ is defined to associate the EI with X-rays that are attenuated by meaningful structures according to the medical purpose of the undergoing exam. In the literature, this step is called ROI selection and, in this paper, we quantify its importance and propose alternative solutions. From the distribution of the gray levels of the pixels $x_{t} \in \Psi$, a value of interest (VOI) $v$ is extracted. Since $v$ has to represent the central tendency of the distribution, it is equal to the median of $\mathbf{q}\left(x_{t}\right), x_{t} \in \Psi$ [3]. Finally, the EI value is computed as follows:

$$
E I=c_{0} g(v)
$$

where $c_{0}^{-1}$ is a constant fixed at $0.01 \mu G y$ according to the norm [1] and $g($. is a calibration function. The value returned by $g(v)$ is the Kerma in the air at the receptor and is expressed in $\mu G y$. The function $g($.$) depends on the \mathrm{X}$ ray system and must be defined in the X-ray standard beam geometry and calibration conditions specified in the norm [1]. Since the final goal is to assess the quality with respect to ideal ALARA dose conditions, a deviation index (DI) is associated with the EI. The optimal exposure is represented by a target EI value $\left(E I_{t}\right)$, and the DI value is formally defined as follows:

$$
D I=10 \log _{10}\left(\frac{E I}{E I_{t}}\right)
$$

where $D I<0$ and $D I>0$ indicate under- and overexposure, respectively.

\subsection{State of the art methods of region of interest definition}

The algorithms for the selection of the ROI suggested in the norm [1] require to initially separate the background, i.e. where X-rays are not attenuated, from the anatomical region. This can be simply achieved by applying to the image $\mathbf{q}$ a threshold defined according to the properties of the system. Therefore, once a subset of anatomical pixel $\Phi \subset \Omega$ is identified, the following methods can be used to define the ROI $\Psi \subset \Phi$. 
Histogram threshold Two thresholds $\bar{\tau}=\left[\tau_{l} ; \tau_{h}\right]$ are applied to the gray levels histogram of $\mathbf{u}\left(x_{t}\right)$ where $x_{t} \in \Phi$. These thresholds are defined as function of two percentile values of the histogram, that, in our experiments, are $1 \%$ and $24 \%$ for $\tau_{l}$ and $\tau_{h}$, respectively. The percentile values are defined according to prior assumptions on the link between intensity values and related physical meaning: the leftmost part of the histogram is associated with strong absorption that is intuitively higher in bone tissues, which are the information of interest. Nevertheless, changes in patient morphotypes, acquisition conditions, presence of metallic objects and so on, influence the gray level distribution and, hence, this way of defining the ROI $\Psi_{\tau}$ may not be consistent across different exams.

Center of the image The ROI can be placed at the center of the image by assuming that the most significant information is centered. The ROI is then, for example, a square or a circle that covers at least $20 \%$ of $\Phi[1]$. In our experiments, we use a square $\Psi_{s}$ that covers $50 \%$ of $\Phi$. Potential problems may occur if the anatomical information of interest is dislocated with respect to the central axis inferred from the indicated collimation limits, e.g. spine in bending exams.

\section{Estimation of the exposure index from local measures associated with landmarks}

\subsection{Anatomical structures detection}

In order to overcome the mentioned drawbacks of state of the art methods, we propose to detect structures of interest in the full-body image. The minimal area bounding boxes that surround the structures of interest are used as ROIs for computing EI values. The detection of such bounding boxes has been widely addressed in the recent literature on medical applications (see [5] and references therein). When 2D images are processed, the bounding box is an oriented rectangle that depends on five parameters: the coordinates of the upper left corner, the width, the height and the orientation. In this paper we do not aim at defining a detection algorithm on full-body X-rays images, but rather at evaluating the application of such techniques to the definition of consistent EI values and, hence, we use the ideal bounding boxes. Nevertheless, in Section 4.2 we study how localization errors influence the EI estimates.

\subsection{Proposed landmark-based approach}

We suggest to associate with an anatomical ROI a cluster of points $l_{j}$ within the structure and, then, get the EI from measures computed in local patches $P_{j}$ centered at these points. In detail, we propose to estimate a local EI measure from the distribution of intensity levels associated with pixels $x_{t} \in P$. We use circular patches of radius equal to 128 pixels. The circle has to be large enough to avoid the measure to be excessively affected by noise and, at the same time, small enough to guarantee the gray level distribution to be approximately mono-modal. 
In this way, the central tendency of a distribution will be a more representative descriptor of the actual amount of signal and, then, of the EI value in a region. Formally, given a set of landmarks $l_{j} \in \mathcal{L}_{r}$, where $\mathcal{L}_{r}$ is the cluster associated with the ROI $\mathcal{A}_{r}$, the corresponding VOIs $v\left(l_{j}\right)$ and, then, the local EI values $e\left(l_{j}\right)$ are computed (Equation 1$)$. The EI value at the ROI $\mathcal{A}_{r}$ is then estimated by using the following weighted sum of local EI values:

$$
E I_{r}=\frac{\sum_{l_{j} \in \mathcal{L}_{r}} \omega\left(l_{j}\right) e\left(l_{j}\right)}{\sum_{l_{j} \in \mathcal{L}_{r}} \omega\left(l_{j}\right)}
$$

where the weights $\omega\left(l_{j}\right)$ assess the accuracy of the measure provided by the landmarks $l_{j} \in \mathcal{L}_{r}$ by giving higher importance to $e\left(l_{j}\right)$ values that are computed from homogeneous gray level distributions because the corresponding VOI, computed as the median, is a good descriptor of the signal level. Formally, we use the entropy of the intensity levels inside the circular patches $P_{j}$ centered at the landmarks $l_{j} \in \mathcal{L}_{r}$ to compute $\omega\left(l_{j}\right)$ and the weights are defined as follows:

$$
\omega\left(l_{j}\right)=\exp \left(\frac{-H\left(l_{j}\right)}{\alpha_{H}}\right)
$$

where $H\left(l_{j}\right)$ is the entropy computed at the patch $P_{j}$ centered at the landmark $l_{j}$ and $\alpha_{H}$ is a constant smoothing parameter set to 2 in our tests. The definition of the landmark positions is out of the scope of this paper, but the effect of detection errors on the measure is quantified in Section 4.2.

\section{Results}

The methods presented in Section 3 are evaluated on EOS full-body images. We will make reference to the histogram-based, to the centered square-based, to the bounding box-based and landmark-based methods with the abbreviations $h R O I$, $s R O I, b b D e t$ and $l D e t$, respectively. On each full-body image, eight regions of interest are studied: head $\left(\mathcal{A}_{1}\right)$, thoracic spine $\left(\mathcal{A}_{2}\right)$, lungs $\left(\mathcal{A}_{3}\right)$, lumbar spine $\left(\mathcal{A}_{4}\right)$, pelvis $\left(\mathcal{A}_{5}\right)$, femur $\left(\mathcal{A}_{6}\right)$, knee $\left(\mathcal{A}_{7}\right)$ and tibia $\left(\mathcal{A}_{8}\right)$. Figure 1a shows an example of manually defined ROIs and the relative EI values are assumed to represent the ground truth. In order to evaluate $h R O I$ and $s R O I$, the full-body images are divided into sub-windows where only one anatomical structure of interest is present because, if the whole anatomical pixel space was taken into account, the estimation errors would clearly be very high. The ideal bounding boxes correspond to the minimal area oriented rectangles that surround these regions, whereas Figure 1b shows an example of manually annotated landmarks. The methods are evaluated according to the DI between the manual and automatic EI values and the standard deviation of DI values $(\sigma(D I))$ over patients in a database associated with each region $\mathcal{A}_{i}$. Similarly to Equation 2, a DI value from the ground truth is computed as follows:

$$
D I=10 \log _{10}\left(\frac{E I}{E I_{g t}}\right)
$$




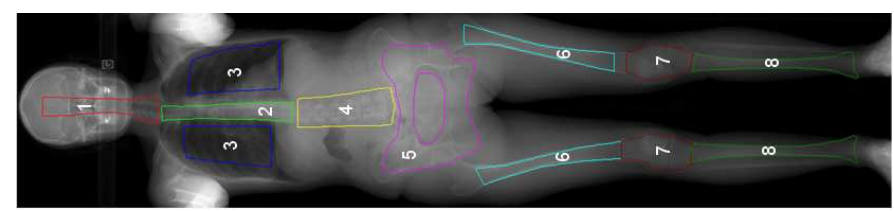

(a)



(b)

Fig. 1. In order to define ground truth EI values, the ROI relative to $\mathcal{A}_{i}, i=1, \ldots, 8$ are manually defined (a). The structures can also be associated with clusters of points (b). The images in the Figures (a) and (b) are not the raw images $\mathbf{q}$ but the post-processed ones for which the grayscale look-up-table is inverted.

where $E I_{g t}$ is the ground truth value associated with a given anatomical ROI. The ideal result is a DI equal to zero, but a margin of error is accepted and different degrees of errors are, thus, considered: $|D I| \in[0,0.25),|D I| \in[0.25,0.5)$, $|D I| \in[0.5,0.75),|D I| \in[0.75,1)$ and $|D I| \in[1,+$ inf $)$ mean, respectively, negligible, low, medium, high and extreme errors. The measure $\sigma(D I)$ quantifies if a given method provides a stable measure over different exams. The methods have been qualitatively compared on a database composed of 82 full-body images by including patients of different ages, and pathological or normal. In the following sections we provide a quantitative evaluation from manually segmented structures on a subset of 6 images that well represents the variability of the data. The parameters specified in Sections 2 and 3 do not change among the tested cases.

\subsection{Comparison of the methods}

Figures $2 \mathrm{a}$ and $2 \mathrm{~b}$ show the root mean-squared-error (RMSE), where the errors are the DI values, and the $\sigma(D I)$ associated with each anatomical structure $\mathcal{A}_{i}$, respectively. The graphs in Figures $2 \mathrm{a}$ and $2 \mathrm{~b}$ do not present $\mathcal{A}_{3}$ because the thresholds $\bar{\tau}$ chosen for the method $h R O I$ are not adapted to the lungs. In other experiments we have focused on the region $\mathcal{A}_{3}$ and, hence, adapted the parameters. The analysis from the associated results is comparable to the one relative to the region $\mathcal{A}_{2}$ that is described in the following comparison.

The method $h R O I$ provides acceptable results only in regions $\mathcal{A}_{4}, \mathcal{A}_{5}$ and $\mathcal{A}_{7}$ as in these areas the signal is quite homogeneous $\left(\mathcal{A}_{4-5}\right)$ or the highest absorption well matches the anatomical structure $\left(\mathcal{A}_{7}\right)$. However, $h R O I$ fails in providing a good exposure indicator in the other regions and, in particular, high errors occur in regions $\mathcal{A}_{2}$ and $\mathcal{A}_{6}$. This happens because the ROI associated with $\mathcal{A}_{2}$ contains the thoracic spine and the soft tissues in the upper part of 


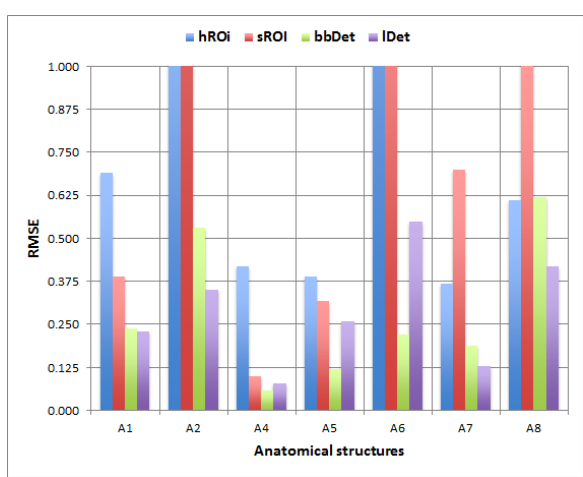

(a)

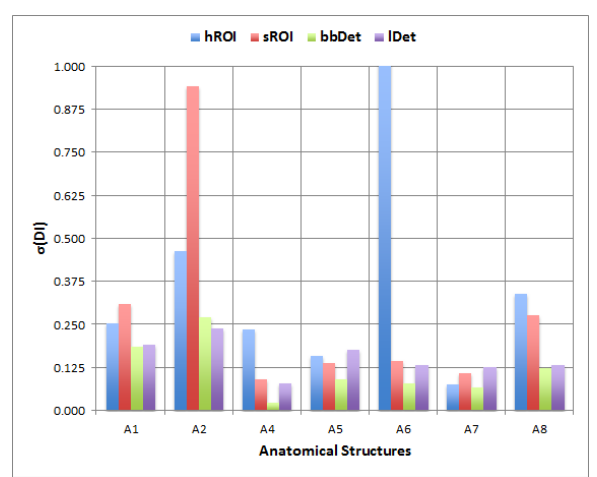

(b)

Fig. 2. Comparison of the methods in terms of: (a) RMSE, (b) $\sigma(D I)$. The graphs show values in the range $[0,1]$.

the lumbar region, while the upper part of the thoracic spine is not included. A similar effect occurs in $\mathcal{A}_{6}$ where the lower part of the pelvis is retained in place of the femurs. Note that the $\sigma(D I)$ values are also high in these regions and, hence, proves that this method does not give consistent EI estimates. By changing $\bar{\tau}$ settings, the results do not improve.

The results with method $s R O I$ are good in $\mathcal{A}_{1}$ and $\mathcal{A}_{5}$ and even negligible errors are observed in $\mathcal{A}_{4}$. In other regions the RMSEs are higher as the anatomical structures are misplaced with respect to the central axis $\left(\mathcal{A}_{6-8}\right)$ or tissues of different densities are included in the ROI $\Psi_{s}\left(\mathcal{A}_{2}\right)$. Nevertheless, the $\sigma(D I)$ values associated with $\mathcal{A}_{6-8}$ are very low, which means that the estimated EI values are consistent. This is however not the case in region $\mathcal{A}_{2}$ and, hence, $s R O I$ is sub-optimal. Moreover, we expect that the performances of this method would decrease in presence of deformed structures (e.g. idiopathic scoliosis) or if the patient is not correctly placed before the acquisition.

The methods bbDet and lDet work very well in all the regions ${ }^{3}$. In detail, bbDet presents RMSE values higher than 0.50 only in regions $\mathcal{A}_{2}$ and $\mathcal{A}_{8}$. In the thoracic spine, the rectangles take into account some of the surrounding pulmonary tissues, which increases the EI value. In region $\mathcal{A}_{8}$, the signal is not very homogeneous, and, hence, the imperfect correspondence between the rectangle and the tibia entails a slight increase of EI value. The method $l D e t$ allows reducing the DI values associated with $\mathcal{A}_{2}$ because by using landmarks the bending of the spine is better respected, whereas in $\mathcal{A}_{6}$ RMSE slightly increases because some soft tissues on the side of the femur are considered in the local measures. However, the $\sigma(D I)$ values obtained with both bbDet and lDet are very low, which proves that in order to obtain consistent EI measures the ROI defini-

\footnotetext{
${ }^{3}$ This is also true for the lungs where the RMSE are equal to 0.22 and 0.13 and $\sigma(D I)$ equal to 0.10 and 0.11 for, respectively, bbDet and $l D e t$.
} 
Table 1. Misplacement in $\mathrm{mm}$ at which the error on the EI values become negligible $(<0.25)$ with bounding boxes, $100 \%, 50 \%$ and $25 \%$ misplaced landmarks for each $\mathcal{A}_{i}$. The higher the value the more robust the method is.

\begin{tabular}{|c||c|c|c|c|c|c|c|c|}
\hline & $\mathcal{A}_{1}$ & $\mathcal{A}_{2}$ & $\mathcal{A}_{3}$ & $\mathcal{A}_{4}$ & $\mathcal{A}_{5}$ & $\mathcal{A}_{6}$ & $\mathcal{A}_{7}$ & $\mathcal{A}_{8}$ \\
\hline bbDet & 10 & $<5$ & $<5$ & 30 & 15 & 5 & 5 & 30 \\
\hline lDet-100\% & 30 & 10 & 10 & 30 & 30 & 15 & 15 & 10 \\
\hline lDet-50\% & 30 & 20 & 15 & 30 & 30 & 30 & 30 & 20 \\
\hline lDet-25\% & 30 & 30 & 20 & 30 & 30 & 30 & 30 & 30 \\
\hline
\end{tabular}

tion in the EI algorithm should be replaced by anatomical structure detection. Moreover, the presence of multiple structures in the field-of-view is implicitly controlled and localization methods can be efficiently implemented [5].

\subsection{Robustness to misplacement errors}

So far only ideal bounding boxes and landmarks have been considered and it remains to quantify the impact of misplaced bounding boxes or landmarks on the estimation of EI values. Therefore, we measure how the EI values change according to simulated localization errors of 5, 10, 15, 20, 25 and $30 \mathrm{~mm}$. For each simulation, the bounding boxes or the landmarks are misplaced with respect to their ground truth positions. The errors for bounding boxes could be induced by mistaken estimation of the position, orientation and size parameters. At first, only errors on the position are taken into account, but changes in orientation or size could probably cause wrong estimation of the EI values as well. As for the landmarks, we evaluate how the performances decrease as the positions of all or part of the landmarks are incorrectly estimated. For each degree of error in distance, 100 simulations are executed and the mean EI values are computed. Then, the DI values are computed with respect to the ideal EI measures, i.e. those obtained from not displaced landmarks or bounding boxes.

The $|D I|$ clearly increases at higher displacement in $\mathrm{mm}$ and Table 1 reports at which distance the errors on EI measure become negligible, i.e. $|D I|<0.25$, according to the method and region $\mathcal{A}_{i}$. In general, the approach based on landmark detection is more robust than the bounding box one. This is due to the redundancy that is introduced in the proposed landmark-based method: if the whole region is misplaced, the central tendency of the gray level distribution will be biased as well, whereas measures from local patches only partially count in the final estimation. As a consequence, if part of the landmarks is correctly located, the EI estimates will be exact within a wide range of spatial error. Quantitatively, all the EI values are correctly estimated if, for example, at least $75 \%$ $(50 \%)$ of landmarks are correctly located within 20 (15) mm, whereas to obtain the same results with $b b$ Det the spatial error has to be inferior to $5 \mathrm{~mm}$. Given these results, we consider unnecessary to simulate errors on the orientation or the size of the bounding boxes that probably would lead to similar conclusions. Finally, Table 1 also highlights that the errors significantly change according to the regions $\mathcal{A}_{i}$, which is coherent with the analysis conducted in Section 4.1. 
The EI values are good in regions $\mathcal{A}_{4-5}$ regardless the detection method and the error degree and, therefore, it will be easy to provide correct measures in these areas. On the other hand, in the chest $\mathcal{A}_{2-3}$ the estimation is more complex given the proximity of structures at low and high density (i.e. the lungs and the thoracic spine) and, hence, the proposed landmark-based method is even more interesting.

\section{Conclusion}

In this paper, we have studied the state of the art methods that are used to estimate EI values from clinical images and then concluded that they may introduce severe errors and are not consistent, especially the histogram-based one. Then, we have shown that more robust and reproducible EI measures can be obtained by relying on anatomical structure detection. Besides, this allows managing the presence of different anatomical structures in the field-of-view, while being efficient. Moreover, we have proposed a landmark-based approach that, by exploiting redundancy of local estimates, is more robust to detection errors than the bounding box-based method. In future works we will present how to automatically detect the landmark clusters. This can be achieved by combining global and local information. A global model encodes the sizes of anatomical structures and the relative landmark positions. The search space is then sequentially narrowed by increasing both efficiency and robustness of the algorithm. The local analysis exploits the positions of salient points associated with peaks of absorption at the detector. The adaptation of the patch shapes to the anatomical structures to which the landmarks are associated is a further perspective.

Acknowledgments Work in part supported by an ANRT grant (2012/0202).

\section{References}

1. IEC 62494-1: Medical electrical equipement - Exposure index of digital X-ray imaging systems - Part 1: Definitions and requirements for general radiography. International Electrotechnical Commission Norm (2008)

2. A. Seibert, D. K. Shelton, E. H. Moore: The standardized exposure index for digital radiography: an opportunity for optimization of radiation dose to the pediatric population. Pediatric Radiology 41, 573-581 (2011)

3. S. J. Shepard, J. Wang, M. Flynn, E. Gingold, L. Goldman, K. Krugh, D. L. Leong, E. Mah, K. Ogden, D. Peck, E. Samei, J. Wang, C. E. Willis: An exposure indicator for digital radiography: AAPM Task Group 116 (Executive Summary). Medical Physics 36, 2898-2914 (2009)

4. S. Illés, S. Somoskeöy: The EOS imaging system and its uses in daily orthopaedic practice. International Orthopaedics 36, 1325-1331 (2012)

5. S. K. Zhou: Discriminative anatomy detection: classification vs regression. Pattern Recognition Letters 43, 25-38 (2014) 